\title{
Simple Modelling of Extracellular Matrix Alignment in Dermal Wound Healing I. Cell Flux Induced Alignment
}

\author{
LUKE OLSEN $^{\mathrm{a}}$, PHILIP K. MAINI ${ }^{\mathrm{a}, *}$, JONATHAN A. SHERRATT ${ }^{\mathrm{b}}$ and BEN MARCHANT ${ }^{\mathrm{a}}$
}

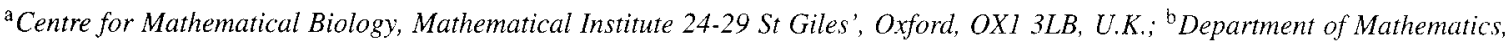
Heriot-Watt University, Edinburgh EHI4 4AS

(Received 21 May 1997; In final form 10 November 1997)

\begin{abstract}
We present a generic model to investigate alignment due to cell movement with specific application to collagen fibre alignment in wound healing. In particular, alignment in two orthogonal directions is considered. Numerical simulations are presented to show how alignment is affected by key parameters in the model. From a travelling wave analysis of a simplified one-dimensional version of the model we derive a first order ordinary differential equation to describe the time evolution of alignment. We conclude that in the wound healing context, faster healing wounds result in more alignment and hence more severe scarring. It is shown how the model can be extended to include orientationdependent kinetics, multiple cell types and several extracellular matrix materials.
\end{abstract}

Keywords: Dynamical reciprocity, travelling waves, scar tissue, haptotaxis

\section{INTRODUCTION AND BIOLOGICAL BACKGROUND}

Dermal wound healing is an extremely complex process. The initial response to a full thickness skin injury is bleeding and the formation of a blood clot, and the progression from this into a contracted scar involves a series of interacting and only partially understood biological processes. Dermal fibroblasts invade the wound space, synthesizing structural proteins including collagen, at the same time as reorganising the existing extracellular matrix (ECM). These fibroblasts also convert reversibly into a contractile phenotype known as myofibroblasts, which are responsible for the process of active 'wound contraction'. Simultaneously, a new capillary network is forming in the wound, and all of these processes are regulated by the synergistic interactions of a large number of peptide growth factors.

Within this highly complex biological situation, detailed mathematical modelling of the entire wound healing process is quite impossible. A number of previous models have been proposed which focus on particular aspects of dermal wound healing. The first of these was the model of (Tranquillo and Murray,

\footnotetext{
*Corresponding Author: Tel: +44 1865 273553; E-mail: maini@maths.ox.ac.uk.
} 
1992), which adapted a mechanochemical framework for developmental patterning to study the role of growth factor regulation in wound contraction. This model was extended by (Olsen et al., 1995; Olsen et al., 1996) to include explicitly the myofibroblast population, leading to putative mechanisms for wound healing disorders such as keloid scarring. (Tracqui et al., 1995) used a different extension of the Tranquillo-Murray model to study the role of nonlinear mechanical properties in wound contraction dynamics. Other workers have developed models for wound healing angiogenesis, building on existing models for this aspect of tumour progression (Pettet et al., 1996; Chaplain and Byrne, 1996).

A key aspect of dermal wound healing which has not been addressed at all in previous modelling is the dynamic alteration of collagen fibre orientation in the ECM during wound healing. The organization of collagen fibres is particularly important, since this underlies many biological and mechanical properties of the tissue, such as tensile strength and extensibility (Fung, 1993). In normal dermis, collagen fibres exhibit a random, 'basket-weave' structure, while scar tissue collagen is generally aligned parallel to the plane of the skin (Harmon et al., 1995; Welch et al., 1990); this is illustrated schematically in Figure 1. This abnormal fibre orientation may cause inferior tissue integrity and strength, and may result in loss of tissue function and deformity around the scar (Rudolph et al., 1992).

Control of fibre alignment is a complex, dynamically varying process. In wound healing, a range of collagens (in addition to many other structural and soluble proteins) are synthesized and secreted by fibroblasts as they migrate into the wound site (Peacock, 1984). These cells also reorganize ECM fibres by a combination of active traction forces and cell locomotion (Eastwood et al., 1994; Ehrlich and Rajaratnam, 1990; Guidry and Grinnell, 1992; Ryan, 1989; Stopak and Harris, 1982); meanwhile, the newly-deposited ECM fibres provide a substrate for cellular movement into the wound site, without which healing cannot occur (McCarthy et al., 1988; Peacock, 1984). This inter-dependent relationship between fibroblasts and ECM has been termed 'dynamic reciprocity' (Clark, 1993). A similar interaction exists between endothelial cells and ECM, and recently Vernon and colleagues have shown that fibroblasts and endothelial cells can form regular geometric patterns cultured on (initially) randomly-oriented fibrillar ECM substrata (Vernon et al., 1992; Vernon and Sage, 1995).

From a modelling viewpoint, such dynamically regulated anisotropy raises major challenges. This is the first of a series of two papers, in which we describe and analyse relatively simple, generic models for the process of ECM alignment during a cellular invasion. Although we will couch our models in the language of wound healing, we do not claim to be modelling any precise details of the wound healing process as such. Rather, our models are generic first attempts to study the alignment process, and our conclusions are consequently general results about this type of dynamically regulated anisotropy.

A number of models have been proposed recently for dynamical alignment in a range of biological

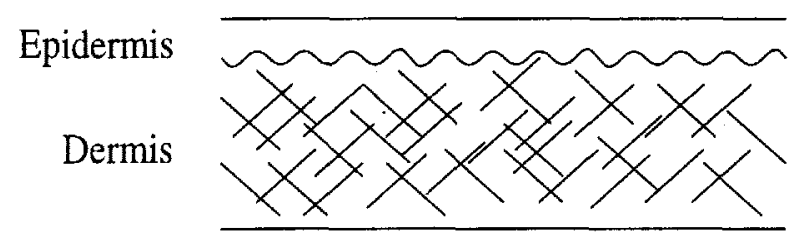

Normal Skin

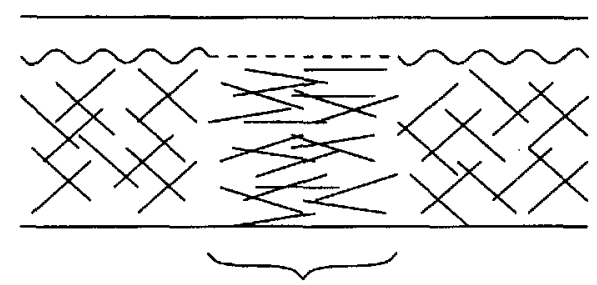

Wound

FIGURE 1 Schematic cross-section through the epidermal and dermal layers of the skin, with ECM fibres represented by short line segments. In normal skin (left) ECM fibres exhibit a 'basket-weave' architecture, whereas in scar tissue (right) there is pronounced fibre alignment parallel to the plane of the skin. 
systems, using both cellular automata and integrodifferential equations. An important prototype biological system is colonies of fibroblasts in vitro, in which cellular orientation is mediated by direct cellcell contacts, giving rise to orientation patterns. This was first modelled by (Edelstein-Keshet and Ermentrout, 1990) and a number of subsequent models have been proposed, typically formulated as integrodifferential equations for the density of cells oriented at a particular angle as a function of space and time (Mogilner and Edelstein-Keshet, 1996).

Another biological alignment system that has been extensively modelled is the intracellular actin filament network, which shows pronounced alignment patterns in response to the local stress field; stress can be either self-generated or externally applied. This was originally modelled by (Sherratt and Lewis, 1993) using a phenomenological spatiotemporal model, enabling in particular the pronounced alignment localised at epithelial wound edges to be studied. In this model, actin alignment is taken to be a function of the ratio of the local, instantaneous, principle components of stress. A more detailed but spatially homogeneous framework was developed by (Civelekoglu and EdelsteinKeshet, 1994), formulated as integro-differential equations for the densities of bound and free actin filaments as a function of orientation and time. This work has recently been extended by (Geigant et al., 1997), focussing on bifurcations from disorder to alignment. A third biological area in which orientation plays a key role is ecological swarming, both of macro-organisms and bacteria; here cellular automata is the most prevelant modelling tool (Stevens, 1995; Deutsch, 1995). (Cook, 1995) and (Grunbaum, 1997) have recently proposed frameworks for reducing integro-differential equations for alignment phenomena to reaction-diffusion-advection equations, which are widely applicable within both ecological and fibroblast culture contexts.

From a computational point of view, the simplest approach is that of cellular automata modelling. However, these models cannot be analysed mathematically and therefore the roles of key parameters can only be determined through an extensive simulation study. The integro-differential equation approach, while more difficult to implement computationally, does offer the possibility of an analytic treatment. However, biologically realistic models are highly nonlinear and model simplifications have been introduced to make the model equations analytically tractable. The approach we adopt in this paper is to devise a model that is both computationally easy to solve and also tractable analytically.

The biological situation described in this paper differs somewhat from these areas, in that there is no intrinsic coupling between ECM orientation at one location and another; rather the only coupling is indirect, via the movement of cells. Nevertheless, a detailed integro-differential equation formulation would be possible, in which the density of ECM fibres would be represented as a function of both space, time and orientation. We deliberately avoid this level of detail for two reasons. Firstly, our aim is to develop a generic model, thus we wish to keep as far as possible to simple, phenomenological rules for alignment. Secondly, we seek to develop models with sufficient mathematical simplicity that wave front solutions ('waves of alignment') can be studied analytically. With these two goals in mind, we represent alignment as a dynamic, reversible conversion between fibres lying in two orthogonal directions.

We begin by introducing a simple, highly generic mathematical model which represents the basic process of cells moving through extracellular matrix. We then extend this isotropic model to account for ECM fibre orientation, and numerical simulations are presented in two space dimensions. Specifically, cell locomotion is assumed to cause fibre alignment, which in turn elicits directional cues for movement via contact guidance. We show that in certain parameter regimes, flux in one of the spatial dimensions may be neglected. Analysis of the resultant simpler one-dimensional model yields insight into the roles of the biological parameters in the alignment process. In Section 3 we discuss various extensions to our model and in Section 4 we address the issue of the permanence of ECM alignment. 


\section{MATHEMATICAL MODELLING}

\subsection{Isotropic Model}

The basic process we are considering is the influx of either fibroblasts or endothelial cells into the wound space. Our modelling is sufficiently generic to apply to either cell type, and henceforth we will just use the term 'cells'. In Section 3, we discuss extending the model to include both cell types. At their most basic, the processes involved in the cellular influx are:

Cells: proliferation, random locomotion (haptokinesis) and directed migration (haptotaxis); each mediated by the ECM.

ECM: biosynthesis and degradation by the cells.

Denoting the cell and ECM densities at position $\underline{r}$ and time $t$ by $n(\underline{r}, t)$ and $m(\underline{r}, t)$, respectively, we have suggested the following (nondimensionalized) mathematical formulation of the above interactions:

$$
\begin{gathered}
\frac{\partial n}{\partial t}+\overbrace{\nabla \cdot[\chi(m) n \nabla m-D(m) \nabla n]}^{\text {haptotaxis and haptokinesis }} \\
=\overbrace{[\alpha(m)-\beta n] n}^{\text {proliferation }} \equiv f(n, m) \\
\frac{\partial m}{\partial t}=\overbrace{\epsilon(1-m) n}^{\text {ECM biosynthesis and degradation }} \\
\equiv g(n, m) .
\end{gathered}
$$

We have previously considered a model of this form (without anisotropy) as a representation of wound healing angiogenesis (Olsen et al., 1997). The parameter $\varepsilon$ is small because ECM remodelling occurs on a significantly longer time scale than cell movement and division (Clark, 1988; Olsen et al., 1997). The ECM equation (2) is an ordinary differential equation, but nevertheless the ECM densities at neighbouring spatial locations are coupled, via the (spatially continuous) cell density.

For convenience, we define the flux vector:

$$
\underline{\mathcal{F}}=\left(\mathcal{F}_{1}, \mathcal{F}_{2}\right)=\chi(m) n \nabla m-D(m) \nabla n .
$$

Note that the division of cell movement into separate directed and random components is an established modelling technique (Murray, 1993). In this application, the two terms act in opposite senses, but the overall direction of cell movement is always into the wound; there is no implication of backwards migration of cells. In some other contexts, haptotaxis is the dominant motility mechanism; an example is tumour invasion which has been the subject of recent modelling work (Perumpanani et al., 1996). However, in the present case, haptotaxis serves merely to modulate the effects of random cell motility.

All parameter values are non-negative, and we specify the following functional forms for the haptotatic, haptokinetic and proliferation terms:

$$
\begin{gathered}
\chi(m)=\frac{\chi_{0}}{\left(\kappa_{\chi}+m\right)^{2}}, D(m)=\frac{D_{0} m}{\kappa_{D}^{2}+m^{2}}, \\
\alpha(m)=\frac{\alpha_{0} m}{\kappa_{\alpha}^{2}+m^{2}} .
\end{gathered}
$$

The haptotactic coefficient is derived from kinetic analysis of a model mechanism for the cell surface receptor-extracellular ligand binding dynamics (Sherratt, 1994). The form of the coefficient of haptokinesis is motivated by specific experimental data (Dickinson and Tranquillo, 1993) and by the intuitive argument that cells cannot move in the absence of a substrate for attachment and that mobility is restricted in very dense matrix. The form of the proliferation rate is motivated by experimental findings (Weinberg and Bell, 1985; Yoshizato et al., 1985) and similar arguments to those above for the coefficient of haptokinesis.

The uniform equilibria of this system are

- $(n, m)=(0, m)$ : a continuum of acellular states with unspecified ECM density, all of which are unstable.

- $(n, m)=(1,1)$ : the equilibrium representing unwounded or normal dermis, in which both cell and ECM density have been scaled to unity; note that $\beta=\alpha(1)$. This is a (globally) stable steady state.

The initial conditions at $t=0$ (the onset of healing) are

- Inside the wound: $(n, m)=\left(0, m_{\text {init }}\right)$ since the wound contains no cells and a low-density ECM substrate $\left(0<m_{\text {init }}<1\right)$. 


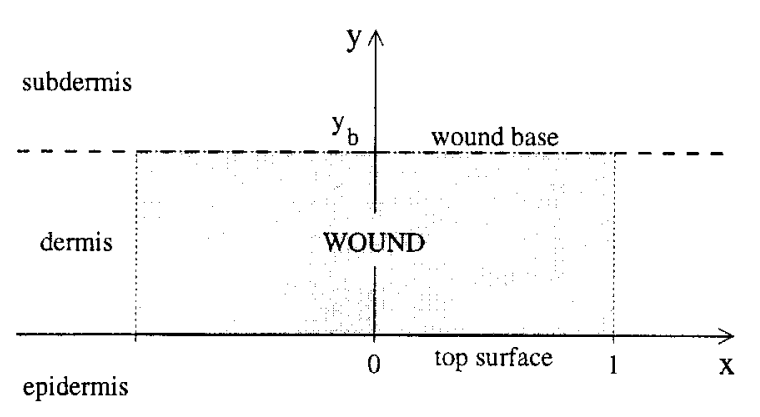

FIGURE 2 Schematic cross-section of the rectangular, fullthickness, dermal wound model, showing the epidermal, dermal and subdermal layers of the skin. The dermis is defined as $-\infty<x<\infty, 0 \leq y \leq y_{b}$ and the wound space is given by $-1<x<1,0 \leq \bar{y} \leq y_{b}$.

- Outside the wound: $(n, m)=(1,1)$ since surrounding tissue is (at least) initially unaffected by the wound.

The domain is restricted to two space dimensions by considering healing dynamics parallel and perpendicular to the plane of the skin, with a rectangular cross-section wound geometry; see Figure 2 . The dermis is the layer $0 \leq y \leq y_{b}$, within which the wound occupies the region $-1<x<1$. By symmetry, equations (1) and (2) need only be defined on the semi-infinite domain $x \geq 0,0 \leq y \leq$ $y_{b}$, with zero-flux at $x=0$, i.e. $\mathcal{F}_{1}(0, y, t)=0$. The other boundary conditions are

- $y=0$ : zero-flux across the wound surface, i.e. $\mathcal{F}_{2}(x, 0, t)=0$.

- $y=y_{b}$ : unwounded steady-state values $(n, m)=$ $(1,1)$ at the wound base.

- $x \rightarrow \infty$ : unwounded steady-state values infinitely far from the wound.

\section{Numerical simulations}

The model equations subject to the above endconditions are solved numerically using a semiimplicit finite difference scheme for the parabolic partial differential equation (1) coupled with a simple forward differentiation formula for the ordinary differential equation (2); see Appendix of (Olsen et al., 1997) for details.

A typical numerical simulation is illustrated in Figure 3, showing wave-like propagation of cells $(n)$ and $\operatorname{ECM}(m)$ moving from the wound peripheries towards the wound centre. Cell levels increase sharply above the normal dermal level in the wavefront (which takes several days to reach the wound centre) and regress much more gradually towards unity in the wave-back. Over this longer time scale (measured in weeks), ECM slowly accumulates behind the wave of cells.

\subsection{Anisotropic Model}

\section{ECM fibre alignment}

We define the proportions of ECM fibres predominantly aligned in the $x$-direction (parallel to the plane of the skin) by $m_{1}$ and in the $y$-direction (perpendicular to the plane of the skin) by $m_{2}$, so that the total ECM density is

$$
m(\underline{r}, t)=m_{1}(\underline{r}, t)+m_{2}(\underline{r}, t) .
$$

This simplistic representation is a more tractable but much less general modelling approach than the alternative of a matrix density that is a continuous function of space, time and orientation. In particular, our representation fails to distinguish between configurations in which the majority of fibres are oriented in one of several discrete directions, and in which orientation is continuously distributed.

ECM fibre alignment between the two orthogonal configurations is also assumed to be a dynamic and reversible process:

$$
m_{1} \stackrel{R_{2}}{\underset{R_{\jmath}}{\rightleftharpoons}} m_{2}
$$

Central to the work in this paper is the following ECM fibre alignment mechanism: it is known that migrating cells remodel local cell-ECM adhesions (Grinnell, 1992; Ingber and Folkman, 1988; Ryan, 1989), causing fibres in the vicinity of a cell to be 'dragged' into an orientation parallel to the direction of cell movement (Eastwood et al., 1994; McCarthy et al., 1988; Repesh et al., 1982). In our model, these arguments suggest that the alignment transition rates, $R_{1}$ and $R_{2}$ in equation (4), should be increasing functions of the magnitudes of the cell 

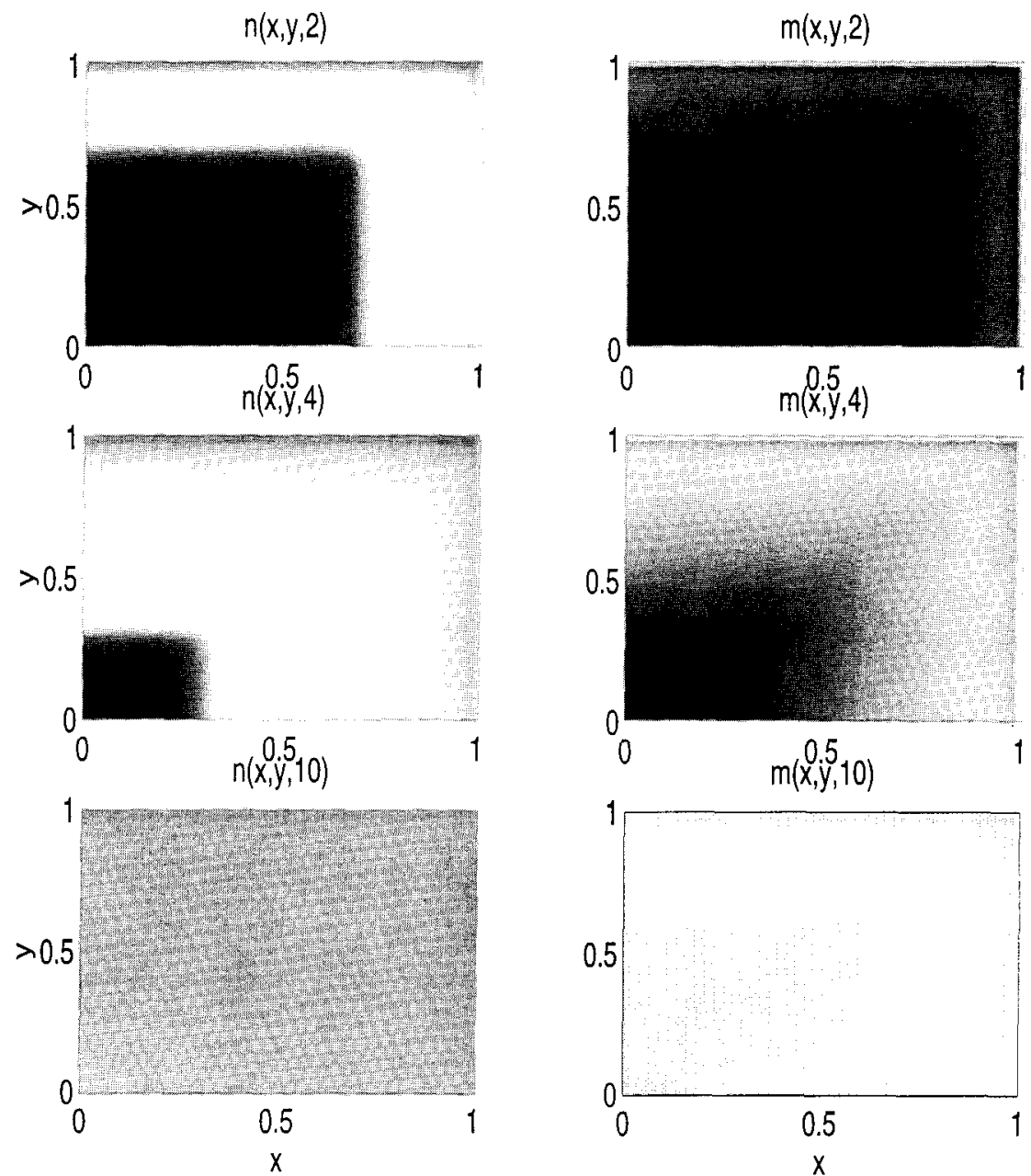

FIGURE 3 Numerical solution of equations (1) and (2) in two spatial dimensions, showing contour plots of cell ( $n$; left) and ECM ( $m$; right) density at times $t=2$ (top), 4 (middle) and 10 (bottom), with end-conditions as in the text. Parameter values are $D_{0}=0.005$, $\kappa_{D}=0.5, \chi_{0}=0.001, \kappa_{\chi}=0.5, \alpha_{0}=1.01, \kappa_{\alpha}=0.1, \beta=1, \epsilon=0.2$ and $m_{\text {init }}=0.1$ and $n_{b}=m_{b}=y_{b}=1$. Only the wound domain $(0 \leq x \leq 1,0 \leq y \leq 1)$ is shown here. The finite difference approximation mesh sizes are $\Delta x=\Delta y=0.02$ and $\Delta t=0.01$. High densities are represented by lighter shading and low densities by darker shading. Top: the early phase of healing, as cells proliferate and migrate into the wound space from the wound margins. Middle: healing is well established, as cells invade the wound space and deposit ECM in the wave-back. Bottom; healing is complete, as cell density recedes from its maximum back towards normal dermal levels and ECM gradually accumulates in the wound space.

flux components $\mathcal{F}_{1}$ and $\mathcal{F}_{2}$, respectively. Without motivation for a more complicated formula, we take

$$
R_{i}=k\left|\mathcal{F}_{i}\right| \quad \text { for } i=1,2
$$

where the alignment rate parameter, $k$, is a positive constant. The immediate implications are that locally in the domain:

- no alignment occurs in the absence of moving cells,
- the rate of alignment increases with the number of cells moving at a fixed speed or with the speed at which a fixed number of cells are moving.

\section{Contact guidance}

An important feedback effect of anisotopy resulting from ECM fibre alignment is the phenomenon of 'contact guidance', in which cells move preferentially 
in the direction of local ECM fibre orientation (Harris et al., 1980; McCarthy et al., 1988; Trinkaus, 1984). In our flux-induced alignment model, this scenario represents two-way positive feedback between cell movement and ECM alignment.

We model contact guidance by assuming that the components of cell flux are proportional to the fractional components of ECM density, so that

$$
\hat{\mathcal{F}}_{i}=\frac{m_{i}}{m} \mathcal{F}_{i} \quad \text { for } i=1,2 .
$$

Hence, $\hat{\mathcal{F}}$ is the anisotropic cell flux vector and $\underline{\mathcal{F}}$ is the isotropic flux, as above.

With the assumption that ECM kinetics (biosynthesis and degradation) are orientation-independent, equations (1) and (2) become

$$
\begin{aligned}
\frac{\partial n}{\partial t}+\frac{\partial \hat{\mathcal{F}}_{1}}{\partial x}+\frac{\partial \hat{\mathcal{F}}_{2}}{\partial y}= & {[\alpha(m)-\beta n] n } \\
\frac{\partial m_{1}}{\partial t}= & k\left(-\left|\hat{\mathcal{F}}_{2}\right| m_{1}+\left|\hat{\mathcal{F}}_{1}\right| m_{2}\right) \\
& +\frac{\epsilon(1-m) n}{2} \\
\frac{\partial m_{2}}{\partial t}= & k\left(\left|\hat{\mathcal{F}}_{2}\right| m_{1}-\left|\hat{\mathcal{F}}_{1}\right| m_{2}\right) \\
& +\frac{\epsilon(1-m) n}{2}
\end{aligned}
$$

Note that equations (2) and (3) are consistent with (7) and (8). The initial conditions on $m_{i}$ are $m_{i}(x, y, 0)=\frac{1}{2} m_{\text {init }}$ inside the wound and $m_{i}(x, y, 0)=\frac{1}{2}$ outside. The boundary conditions are $m_{i}(x \rightarrow \infty, y, t)=m_{i}(x, y b, t)=\frac{1}{2}$.

\section{Steady states}

Since the cell fluxes and kinetics terms (i.e. the functions $f$ and $g$ ) are zero at the stable equilibrium where $n=m=1$, the values of $m_{i}$ are not determined and, in particular, may vary with position. Equation (3) imposes the constraint

$$
m_{1}(x, y, t \rightarrow \infty)+m_{2}(x, y, t \rightarrow \infty) \equiv 1 .
$$

The analysis in this paper is focussed towards gaining insight into how the degree of alignment depends on the biological processes and parameters within the model. Once $m_{1}$ (say) is known at steady state, $m_{2}$ is given by (9). A combination of analytical approximations and numerical simulations (details omitted) suggests that there are no stable spatiallyvarying equilibria.

\section{Limiting cases}

As the alignment rate parameter $k \rightarrow 0$, we retrieve the isotropic model given by equations (1) and (2), giving no overall anisotropy at any time.

In the limit as $k \rightarrow \infty$, equations (7) and (8), together with (5), imply that the alignment and flux ratios are equal:

$$
\frac{m_{1}}{m_{2}} \sim\left|\frac{\hat{\mathcal{F}}_{1}}{\hat{\mathcal{F}}_{2}}\right|,
$$

which in the case of anisotropic (contact guidance) cell flux, implies either $\left|\mathcal{F}_{1}\right|=\left|\mathcal{F}_{2}\right|, m_{1}=0$ or $m_{2}=0$.

\section{Numerical simulations}

Equations (6)-(8) and associated end-conditions are solved using the same numerical methods as in the isotropic model, with the extra flux-induced alignment terms treated (explicitly) as kinetic terms in equations (7) and (8).

Typical simulations of ECM density and fibre orientation during wound healing are shown in Figure 4, for two different values of the alignment rate parameter $k$; no experimental data currently exists to estimate the biological value of this parameter. The following features are observed:

- Cell density $(n)$ and total ECM density $(m)$ are indistinguishable from those in Figure 3 (not shown), for both values of $k$.

- The overall extent of ECM alignment is strongly dependent on $k$.

- For a relatively low value of $k$ (Figure 4; left), alignment is barely discernible (except near the wound margins $x=1$ and $y=1$ ).

- For a larger value of $k$ (Figure 4; right), alignment is marked throughout most of the domain. 

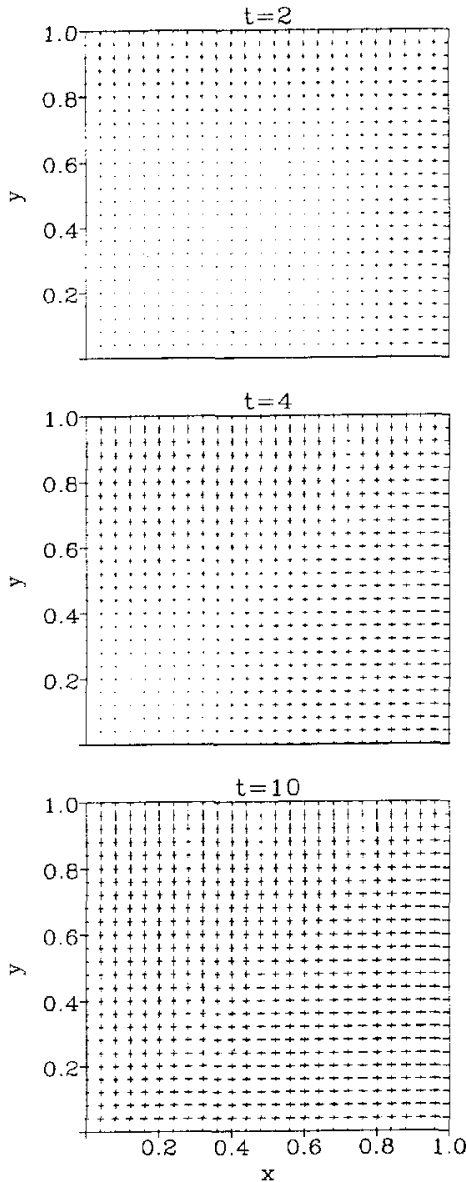
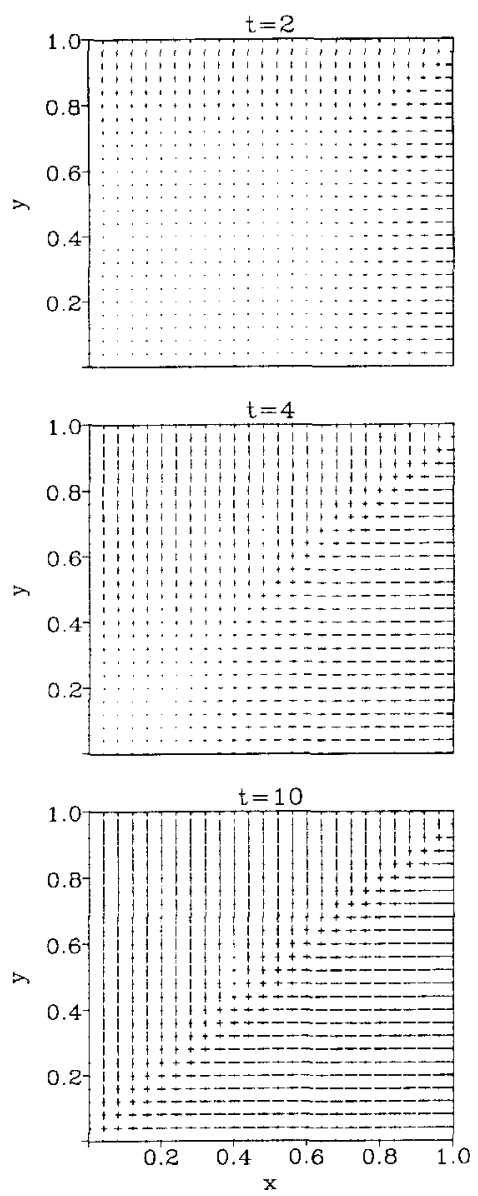

FIGURE 4 Numerical simulations of equations (6)-(8) illustrating $m_{1}(x, y, t)$ and $m_{2}(x, y, t)$ at evenly-spaced points throughout the wound domain $0 \leq x<1,0<y<1$, at times $t=2,4$ and 10 . Horizontal and vertical dashes measure $m_{1}$ and $m_{2}$, respectively, so that the size of the crosses (" + -signs) is the total ECM density $(m)$ and the degree of axial eccentricity of a cross represents the extent of local ECM fibre alignment. Parameter values are: $D_{0}=0.01 K_{D}=0.5, \chi_{0}=0.002, \kappa_{x}=0.5, \alpha_{0}=1.01, \kappa_{\alpha}=0.1, \beta=1$ $\epsilon=0.2$ and $m_{\text {nit }}=0.1$, with end-conditions as in the text. The values of $D_{0}$ and $\chi_{0}$ are double those in Figure 3 in order to maintain (roughly) the same magnitude of flux coefficients as in the isotropic-flux model (i.e. without contact-guidance). End-conditions are as in the text. Left column: the value of the alignment rate parameter, $k=10$, elicits only slight alignment, although anisotropic effects are greater around the wound periphery (near $x=1$ and $y=y_{b}$ ). Right column; with a higher alignment rate parameter, $k=50$, a considerably more pronounced orientation pattern is observed (see text for explanations).

- Alignment effects are more pronounced near the wound margins.

- No net alignment is apparent within a narrow neighbourhood of the line $y=x$, and away from this line, most ECM fibres become aligned in the direction of the dominant cell flux. In this sense, there is $x-y$ symmetry in the wound.

- Over considerably longer times than those shown in Figure 4 (bottom row), the ECM orientation profiles within the wound do not change significantly.
The numerical simulations suggest that in most of the wound domain, the behaviour of the system is approximately one-dimensional. For the purposes of tractable analysis, therefore, we next consider the 1 -D analogue of this flux-induced alignment model.

\subsection{Analysis of the 1-D Model}

We consider the dynamics in the $x>y$ portion of the wound by neglecting the cell flux in the $y$-direction, $\mathcal{F}_{2}$, from equations (6)-(8) (from Figure 4 , it can be 
seen that this approximation increases in accuracy as $k$ increases), giving the following 1-D model:

$$
\begin{aligned}
\frac{\partial n}{\partial t}+\frac{\partial \hat{\mathcal{F}}_{1}}{\partial x} & =[\alpha(m)-\beta n] n \\
\frac{\partial m_{1}}{\partial t} & =k\left|\hat{\mathcal{F}}_{1}\right| m_{2}+\frac{\epsilon(1-m) n}{2} \\
\frac{\partial m_{2}}{\partial t} & =-k\left|\hat{\mathcal{F}}_{1}\right| m_{2}+\frac{\epsilon(1-m) n}{2},
\end{aligned}
$$

where

$$
\hat{\mathcal{F}}_{1}=\frac{m_{1}}{m}\left[\chi(m) n \frac{\partial m}{\partial x}-D(m) \frac{\partial n}{\partial x}\right] .
$$

The end conditions at $t=0, x=0$ and as $x \rightarrow \infty$ are as above. It is entirely consistent to study alignment within the context of a one-dimensional model, meaning simply that the degree of alignment is a function of one of the spatial coordinates only. For example, in a long thin ('slash') wound, there will typically be preferential ECM alignment orthogonal to the wound, but to an extent that depends only on distance away from the line of the wound.

\section{Numerical simulations}

Equations (10)-(12) are solved numerically using a semi-implicit finite difference method (based on the Crank-Nicholson scheme) which is similar to that used for the analogous 2-D simulations.

Typical numerical simulations are presented in Figure 5, illustrating the profiles of cell density $(n)$, total ECM density $(m)$ and the aligned component of ECM density in the $x$-direction $\left(m_{1}\right)$, at several successive time intervals. As in 2-D, the key observations are:

- Profiles of cells and total ECM are not significantly different from those in the isotropic ( $k=$ 0) model (not shown).

- ECM alignment occurs in the wound behind the advancing wave of cells.

- Alignment effects are more pronounced near the wound margin $(x=1)$.

Also, the ECM does not become aligned (i.e. $m_{1}=$ $0.5)$ at the wound centre $(x=0)$ due to the zero-flux boundary condition.

In most of the wound space $(0 \leq x \leq 1)$, the steady-state value of $m_{1}$ is roughly constant and greater than 0.5 when $k>0$. Defining this steadystate value of $m_{1}$ in the wound space as $\phi$, the aim of the ensuing analysis is to determine how $\phi$ depends on $k$ and on other biological parameters. Biologically, the greater the value of $\phi$ above 0.5 , the greater the degree of permanent ECM alignment in the healed wound, and hence, the greater the severity of scarring.

\section{Travelling wave analysis}

Numerical simulations such as those in Figure 5 suggest that the model may possess travelling wave solutions: that is, the solutions for $n(x, t)$ and $m(x, t)$
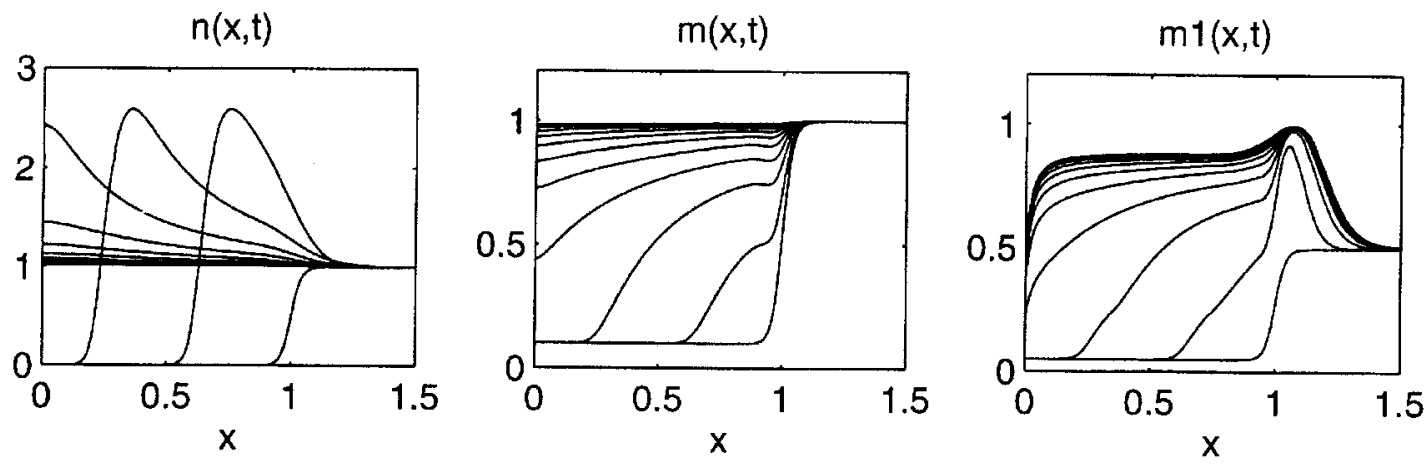

FIGURE 5 Numerical solution of equations (10)-(12) showing profiles of cell density $(m)$, total ECM density $(n)$ and the aligned component of ECM density in the $x$-direction $\left(m_{1}\right)$, at times $t=0,2,4, \ldots, 20$. Arrows indicate the direction of the wave-like evolution of these profiles, toward $x=0$ (the wound centre). The wound space is $0 \leq x \leq 1$, and $x>1$ represents unwounded dermis. Parameter values are as in Figure 4, with the alignment rate parameter $k=50$. The long-time profiles do not differ significantly from those at time $t=20$. 
may develop (from the initial conditions) into wave profiles which propagate with constant speed and form. This hypothesis is considered in this section, in order to investigate the dependence of the wave speed on the key biological parameters.

We begin by seeking travelling wave solutions of equations (10)-(12) of the form

$$
\begin{array}{cl}
N(z)=n(x, t), & M_{1}(z)=m_{1}(x, t), \\
M_{2}(z)=m_{2}(x, t) & \text { and } \quad M=M_{1}+M_{2},
\end{array}
$$

where $z=x+c t$ is the travelling wave coordinate and $c>0$ is the wave speed. Under this transformation, equations (10)-(12) become:

$$
\begin{aligned}
c N^{\prime}+\hat{\mathcal{F}}_{1}^{\prime} & =[\alpha(M)-\beta N] N \\
c M_{1}^{\prime} & =k\left|\hat{\mathcal{F}}_{1}\right| M_{2}+\frac{\epsilon(1-M) N}{2} \\
c M_{2}^{\prime} & =-k\left|\hat{\mathcal{F}}_{1}\right| M_{2}+\frac{\epsilon(1-M) N}{2}
\end{aligned}
$$

where

$$
\hat{\mathcal{F}}_{1}=\frac{M_{1}}{M}\left[\chi(M) N M^{\prime}-D(M) N^{\prime}\right]
$$

and ' denotes $d / d z$.

A standard linear analysis of equations (13)-(15), both far ahead of and far behind the wave, reveals that stable travelling wave solutions may exist - although we cannot prove this - and that the minimum permissible wave speed of such solutions is given by

$$
c_{\min }=\sqrt{2 D\left(m_{\text {init }}\right) \alpha\left(m_{\text {init }}\right)} .
$$

Note that the theoretical minimum wave speed does not depend on the alignment rate parameter, $k$. This is counter-intuitive, in that the contact-guidance flux feedback effect should accelerate wound healing, with greater effect as the alignment response becomes faster. Hence, we expect the wave speed $c$ to increase with $k$. In addition, we certainly expect $\phi$ (the steady-state value of $m_{1}$ ) to increase with $k$. Below, we use numerical simulations similar to those in Figure 5 to resolve these issues.

Figure 6 reveals that these expectations are borne out: $\phi$ is more sensitive than $c$ to changes in $k$, however, so $\phi$ also increases with $c$. Because the linear analysis predicts that $c$ does not depend on $k$ (see above), the weak dependence of $c$ on $k$ is not surprising - it is due to nonlinearities in equations (13)-(15). Taken together, these results suggest that faster healing is correlated with greater alignment and more marked scarring.

\section{Quantification of final ECM alignment}

The structure of the travelling wave equations (13)-(15) may be exploited to obtain insight into
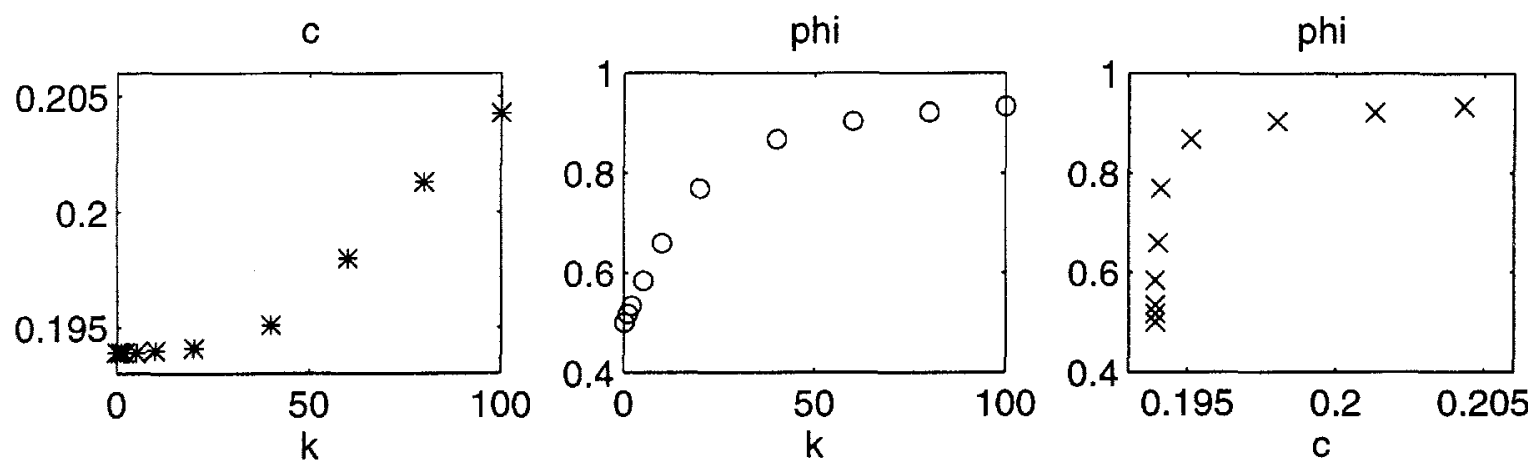

FIGURE 6 Numerical solutions of equations $(10)-(12)$ for $k=0,1,2,5,10,20,40,60,80$ and 100 from which the wave speed $c$ (left: *) and steady-state value of $m_{1}$ (i.e. $\phi$; middle: 0 ) are calculated. Also shown is the relationship between $\phi$ and $c$ (right: $x$ ), Parameter values (except $k$ ) as in Figure 4. An extended spatial domain is used in these simulations to allow long-time evolution of the wave speed. The theoretical minimum wave speed, $c_{\min } \approx 0.197$, is predicted by linear analysis of travelling wave solutions (see text). These results show that the wave speed $(c)$ and extent of scarring (given by $\phi$ ) both increase with the alignment rate parameter $(k)$, the latter relationship being the more sensitive. Note the very fine resolution of the axis for $c$, which confirms our result in the main text that $c$ is approximately independent of $k$ 
the relationships between the model variables far behind the wave, and hence, into the value of $\phi$.

To begin our analysis, we define the quantity $A \equiv M_{1}-M_{2}$, so that $A=0$ implies no overall alignment and $A=M$ represents maximal alignment. Subtracting equation (15) from (14) yields

$$
c A^{\prime}=k\left|\hat{\mathcal{F}}_{1}\right|(M-A) \text {. }
$$

To facilitate our analysis, we neglect the haptotactic contribution (coefficient $\chi$ ) to the cell flux at present, since it is the haptokinetic term (coefficient $D$ ) that has been shown to drive wound healing in this model (see above). In this case, the above ODE simplifies to

$$
A^{\prime}=\frac{k D(M)\left|N^{\prime}\right|\left(M^{2}-A^{2}\right)}{2 c M} .
$$

We require the value of $A$ as $z \rightarrow \infty$, since $\phi=$ $\frac{1}{2}(A+1)$ in this limit (i.e. far behind the wave). It can be shown, using perturbation theory based on the structure of the travelling wave solutions (as in Figure 5), that

$$
N(z)=\alpha[M(z)] / \beta
$$

is a reasonable leading-order approximation for the solutions in the wave-back for small $\epsilon$, as is the case here (see Olsen et al., 1997, for details). Then, using the fact that $M(z)$ is a monotonic increasing function and dividing through the above ODE by $M^{\prime}$, we derive the non-autonomous initial value problem (IVP)

$$
\frac{d A}{d M}=h(M)\left(M^{2}-A^{2}\right)
$$

where

$h(M)=\frac{k D(M)}{2 c M}\left|\frac{d \alpha}{d M}\right|=\frac{k D_{0} \alpha_{0}\left|\kappa_{\alpha}^{2}-M^{2}\right|}{2 c\left(\kappa_{D}^{2}+M^{2}\right)\left(\kappa_{\alpha}^{2}+M^{2}\right)^{2}}$, together the initial condition $A\left(m_{\text {init }}\right)=0$ since there is no ECM alignment ahead of the wave. Here, as in the simulations, we have fixed the value of $\beta$ at 1. This IVP, for $A$ as a function of $M$, yields the value of $\phi$ via the formula $A(1)=2 \phi-1$.

The complicated form of the coefficient $h(M)$ implies that the IVP (17) cannot be solved exactly (in closed form). However, the fact that the parameter grouping

$$
P=\frac{k D_{0} \alpha_{0}}{2 c}
$$

appears in (17) implies that $\phi$ will depend on $P$. In addition, if wave solutions propagate at their minimum speed, $c_{\min }$ given by (16), then

$$
P=\frac{k}{2} \sqrt{\frac{D_{0} \alpha_{0}}{2}}
$$

The results shown in Figure 6 (left) suggest that this is a fairly accurate assumption.

A further simplification can be obtained by approximating the cell proliferation function as follows:

$$
\alpha(M)=\frac{\alpha_{0} M}{\kappa_{\alpha}^{2}+M^{2}} \approx \frac{\alpha_{0}}{M} .
$$

This is appropriate because $\kappa_{\alpha} \ll 1$ (see Figure 4 legend) and is valid for values of $M$ greater than $\kappa_{\alpha}$. Here, we require solutions of (17) as $M \rightarrow 1$, where the above approximation is a very good fit. In this case, (17) becomes

$$
\frac{d A}{d M}=h_{\text {approx }}(M)\left(M^{2}-A^{2}\right)
$$

where

$$
h_{\text {approx }}(M)=\frac{k D_{0} \alpha_{0}}{2 c\left(\kappa_{D}^{2}+M^{2}\right) M^{2}} \approx h(M) .
$$

This simpler problem is still not solvable exactly, due to the polynomial denominator of $h_{\text {approx }}(M)$ arising from the form of $D(M)$, so the IVP (18) must be solved numerically.

Figure 7 shows the predicted approximations for $\phi$ as $k$ varies, given by numerical solutions of both the IVPs (17) and (18). It is clear that these relatively simple IVPs yield quantitatively accurate approximations to the real values of $\phi$ obtained from numerical solutions of the full PDE system (10)-(12). In fact, the dependence of $\phi$ on $k$ is also captured by a simple, saturating function of the form

$$
\bar{\phi}(k)=\frac{1}{2}\left(1+\frac{k}{K_{1 / 2}+k}\right)
$$




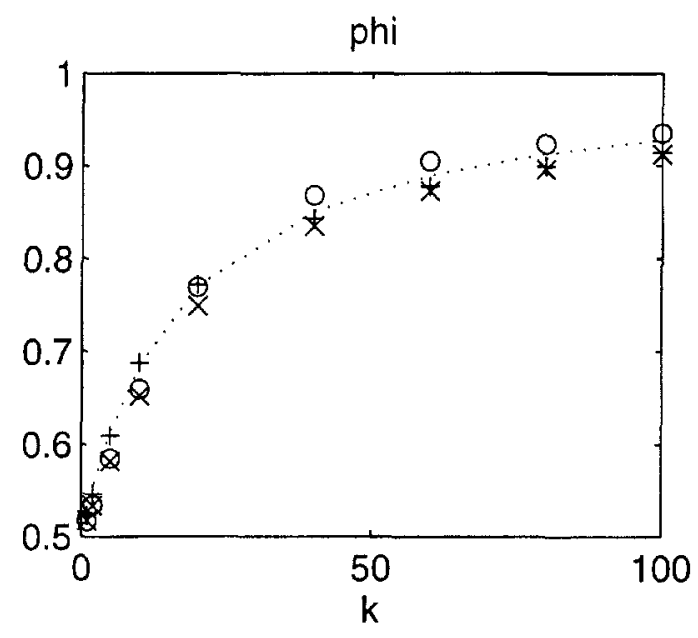

FIGURE 7 Numerical solutions of the model partial differential equations (10)-(12) (and of the approximating ordinary differential equations (17) and (18)) for $k=1,2,5,10,20,40,60$. 80 and 100 , from which the values of $\phi$ are calculated, given by circles $(0)$, crosses $(x)$ and plus-signs $(+)$, respectively. Other parameter values and theoretically-predicted wave speed are as in Figures 4 and 6, respectively. The initial value problem (17) and a simpler approximation (18) both yield good quantitative estimates of the values of $\phi$ for all $k$ in the range shown, using $c_{\min }=0.197$ in (17) and (18). The dotted curve is the simple saturating function $\bar{\phi}(k)=\frac{1}{2}(1+k /(17+k))$, which also gives a close approximation to the PDE results for $\phi$. This figure shows that the dependence of $\phi$ on $k$ is a convex, monotonic increasing function with $\phi=\frac{1}{2}$ when $k=0$ and $\phi \rightarrow 1$ as $k \rightarrow \infty$. as captured by the solutions of the IVPs (17) and (18).

where $K_{1 / 2}$ is a positive constant. The solutions $\phi(k)$ derived from the IVPs (17) and (18) presumably have a similar qualitative form as $\bar{\phi}$.

Finally, since the parameter grouping $P$ is directly proportional to $k$, we deduce that $\phi$ (the steady state value of $m_{1}$ ) varies with $P$ in the manner shown in Figure 7. Thus, in conclusion, $\phi$ is a monotonic increasing function of the alignment rate parameter $k$, of the cell haptokinesis (random motility) coefficient $D_{0}$ and of the cell proliferation coefficient $\alpha_{0}$.

\section{Roles of Other Parameters}

To date, we have analysed the effects of the cell proliferation, cell haptokinesis (random, or unbiased movement) and alignment rate parameters on the degree of steady-state alignment. Here, we briefly assess the sensitivity to changes in the cell haptotaxis (biased movement) and ECM kinetics (synthesis and degradation) parameters using numerical simulations, since the analysis above does not account for these effects.

Numerical simulations reveal that increased haptotactic sensitivity (as measured by $\chi_{0}$ ) results in greater ECM alignment, as shown in Figure 8. This is explained by a qualitative mathematical analysis of the two cell fluxes - haptotaxis and haptokinesis - in the wave profiles (see Figure 5, for example). Just ahead of the wave, the negative haptotactic flux (equal to $-D(m) \partial m / \partial x$ ) dominates the positive haptotactic flux (equal to $\chi(m) n \partial m / \partial x$ ): this is necessary for equation (10) to be of parabolic type and to admit travelling wave solutions in the direction towards the wound centre. Behind the wave, however, both fluxes are positive (since $\partial n / \partial x$ is negative in the wave-back and $\partial m / \partial x$ is always non-negative), so haptotaxis enhances the total cell flux, leading to greater flux-induced alignment. Note that the haptotactic flux has a greater effect on alignment in the wave-back than in the wave-front, since most ECM accumulation occurs behind the wave.

In addition, Figure 8 shows that ECM alignment is inhibited by faster ECM kinetics (as measured by $\epsilon)$. Biologically, this implies that accelerated ECM deposition and degradation (i.e. faster ECM remodelling) results in less fibre alignment at steady-state.

\section{MODEL EXTENSIONS}

\section{Orientation-Dependent Kinetics}

So far we have assumed that the ECM kinetics are orientation-independent. This means that when it is secreted half of the ECM is orientated in each direction. However, there is a tendency for fibroblasts to lay down ECM fibres in the direction of their motion (Birk and Trelstad, 1986). We now incorporate this idea into the ECM kinetics by writing:

$$
g_{i}(n, m)=(1-m)\left(k_{2} \hat{\mathcal{F}}_{i} n+n\right)
$$

There are now two types of biosynthesis, that in the direction of fibroblast movement and that independent of it. The parameter $k_{2}$ represents the 
phi

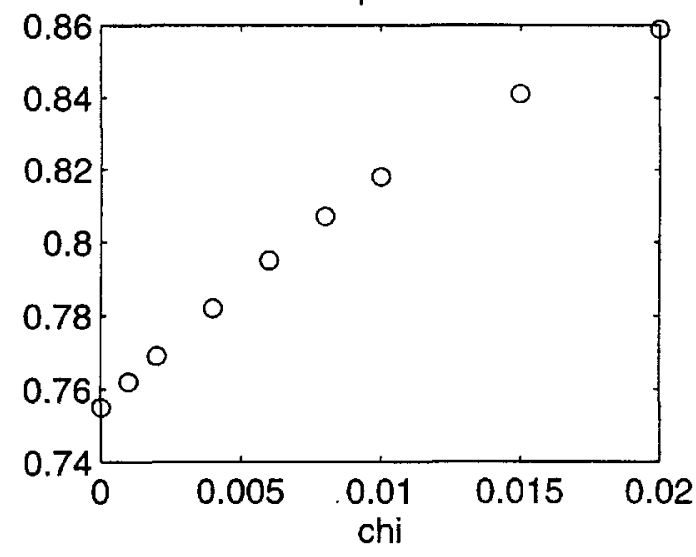

phi

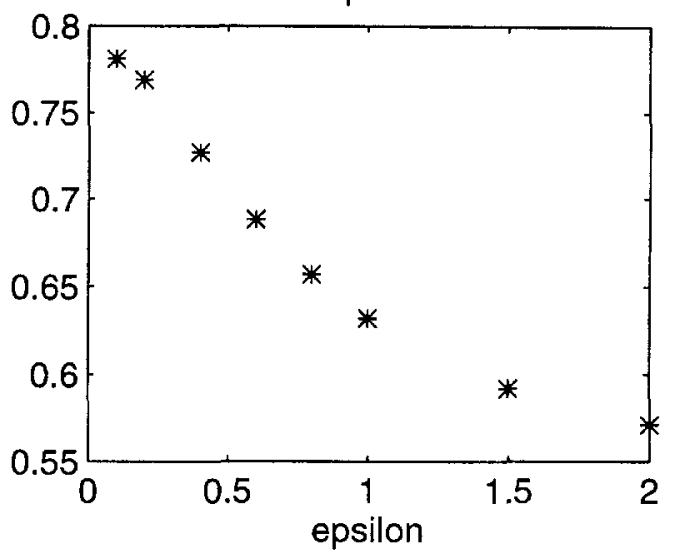

FIGURE 8 Numerical solutions of the equations (10)-(12), from which $\phi$ is measured, as the haptotactic sensitivity parameter $\chi_{0}$ (left; 0 ) and the ECM kinetics parameter $\epsilon$ (right; $*$ ) are varied. Parameter values as in Figure 4 , except $k=20$. These results show that the extent of steady-state ECM fibre alignment increases with $\chi_{0}$ and decreases with $\epsilon$.
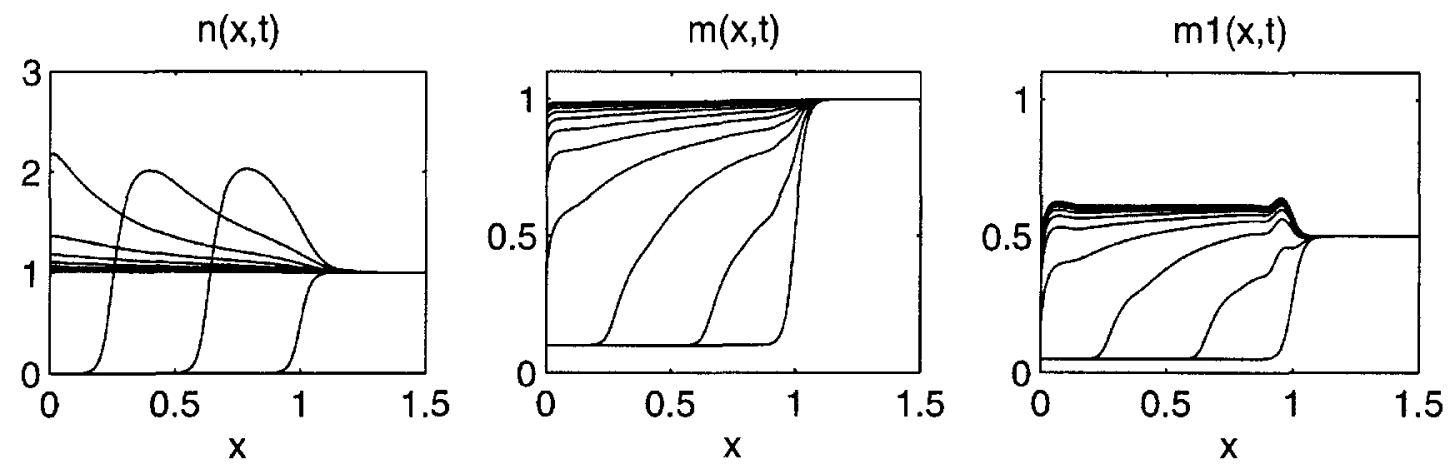

FIGURE 9 Numerical solution of equations (10)-(12) incorporating the orientation-dependent kinetics term (19), showing profiles of cell density $(m)$, total ECM density $(n)$ and the aligned component of ECM density in the $x$-direction $\left(m_{1}\right)$, at times $t=0,2,4, \ldots, 20$. The wound space is $0 \leq x \leq 1$, and $x>1$ represents unwounded dermis. Parameter values are as in Figure 4 , with the flux-induced alignment parameter $k=0$ and the orientation-dependent biosynthesis parameter $k_{2}=10$. The long-time profiles do not differ significantly from those at time $t=20$.

ratio of biosynthesis by each method. Note that if all the biosynthesis is dependent on fibroblast direction, then the matrix becomes 100 percent aligned in the majority of the domain, where the flux is only in one direction.

We substitute the new kinetic terms (19) into equations (10)-(12) and solve them numerically using a similar scheme to that in Section 2.3. We consider analogous simulations to those illustrated in Figure 8 but with $k=0$ so that all the alignment is caused by the kinetics terms. We find that the solutions are very similar to those for the flux-induced alignment model. The profiles of cells and ECM are not significantly different and there is still pronounced alignment near the wound margin $(x=1$ in Figure 9). The amount of alignment within the wound increases with $k_{2}$, as illustrated in Figure 10. Therefore, in this model framework, flux-induced alignment, alignment due to orientation-dependent kinetics or a combination of each of these appear to be equally valid mechanisms for the alignment of ECM fibres. A more detailed modelling framework would be required to distinguish between these mechanisms. 


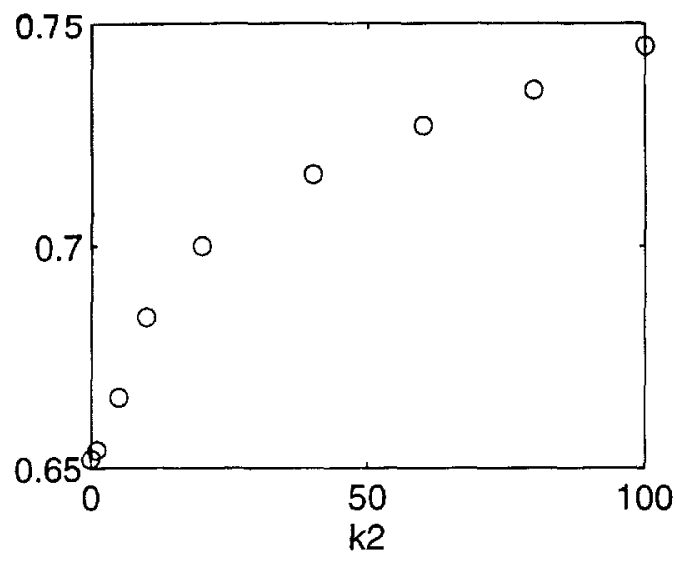

FIGURE 10 Numerical solutions of the model partial differential equations (10)-(12) incorporating the orientatjon-dependent kinetics term (19), from which the values of $\phi$ are measured Parameter values are as in Figure 9 except $k=10$ and $k_{2}=1$, $2,5,10,20,40,60,80$ and 100 .

\section{Multiple Cell Types}

Here, we outline an extension to the basic model of Section 2 in which the dynamics of more than one cell type is explicitly modelled. This scenario is motivated by the involvement of at least two key cell types - fibroblasts and endothelial cells - in wound healing. In this case, each cell type is represented by a continuum variable governed by an equation of the form (6). We assume that each cell type exhibits ECM-mediated motility and proliferation, and that each cell type synthesizes and degrades the ECM.

Crucially, multiple cell fluxes cause greater ECM alignment than a single flux, since the distinct fluxes combine additively on the right-hand sides of the analogues of equations (7) and (8). This effect may be modulated, however, by the accelerated ECM accumulation resulting from the involvement of multiple cell types. Numerical simulations reveal that the net effect on ECM fibre alignment depends on the various parameter values (details not shown).

\section{Multiple ECM Types}

The other obvious model extension accounts for more than one ECM variable. Thus far, the ECM has been modelled on fibrillar collagen which is the predominant protein in normal dermis and in scar tissue. Other ECM constituents also perform important roles in wound healing: for example, elastin fibres confer extensibility and strength to unwounded dermis and fibronectin is a key mediator of cell-collagen interactions and is crucial in wound healing.

Within our model, each ECM variable is assumed to regulate ECM motility and proliferation as in equation (6), and has dynamics (synthesis, degradation and flux-induced alignment) encapsulated by equations of the same form as (7) and (8). Thus, each ECM type becomes permanently aligned by the cell flux, to a degree that depends not only on the specific alignment rate parameter (each ECM type has a distinct $k$ ), but also on the contributions of every ECM type in the cell flux. The analysis of Section 2.3 is no longer possible, but numerical simulations confirm that flux-induced ECM alignment does occur, with the steady-state alignment depending on the parameters as expected.

\section{THE PERMANENCE OF ECM ALIGNMENT}

In our model, no further ECM alignment occurs far behind the wave of proliferating cells, once the total ECM density $(m)$ has reached unity (recall that $m=$ 1 implies $g=0$ ). In the sense that ECM alignment is permanent, the model therefore predicts a 'static' equilibrium after healing, in which the ECM is neither synthesized nor degraded. In some wounds, however, ECM remodelling continues for several years after injury, during which time fibre alignment may occur (Clark, 1993; McPherson and Piez, 1988; Shah et al., 1992). This may be described as a 'dynamic' equilibrium, in which both ECM synthesis and degradation occur, but at equal rates so that the total ECM density is conserved. Biologically, ECM reorganization cannot occur when in static equilibrium, since dynamic remodelling of the cross-linking between ECM fibres is required (Ingber and Folkman, 1988; Mast, 1992). 
To investigate these details, we may regard ECM biosynthesis and degradation as separate functions, given by the rates $\epsilon n$ and $-\epsilon m n$ respectively. For the orthogonal ECM components, $m_{i}$, ECM biosynthesis is assumed to be independent of local ECM orientation, whereas the rates of degradation are proportional to $m_{i}$. In this case, the (1-D) model equations (11) and (12) become:

$$
\begin{aligned}
& \frac{\partial m_{1}}{\partial t}=k\left|\hat{\mathcal{F}}_{1}\right| m_{2}+\epsilon\left(\frac{1}{2}-m_{1}\right) n \\
& \frac{\partial m_{2}}{\partial t}=-k\left|\hat{\mathcal{F}}_{1}\right| m_{2}+\epsilon\left(\frac{1}{2}-m_{2}\right) n .
\end{aligned}
$$

Note that $g(m, n)=\epsilon(1-m) n$ by adding these equations, as previously. The key difference here is that the steady-state values of $m_{1}$ and $m_{2}$ are now fixed - both are equal to $\frac{1}{2}$, so that ECM alignment is no longer permanent. Thus, the ECM is now in dynamic equilibrium, since remodelling will occur (i.e. the $m_{i}$ will change) when the total ECM density $(m)$ has attained its equilibrium value (unity), resulting in an isotropic configuration.

The extent to which the ECM in normal scar tissue is in dynamic equilibrium is unclear. This is partly because ECM biosynthetic and proteolytic activity in a wound would have to be monitored over a time scale of months or years to assess the permanence of ECM fibre alignment. Evidence suggests, however, that the ECM gradually becomes quiescent, or static with time (Mast, 1992). Thus, alignment may be only partially corrected.
ECM biosynthesis and degradation can be modelled as separate processes whilst retaining a continuum of equilibria for $m_{1}$ and $m_{2}$ (as in the original model of Section 2) by assuming that as the total ECM density $(m)$ approaches steady state, these processes are downregulated via density-dependent negative feedback - this mechanism is suggested by biological data (Clark, 1993). For example:

$$
\begin{aligned}
& \frac{\partial m_{1}}{\partial t}=k\left|\hat{\mathcal{F}}_{1}\right| m_{2}+\epsilon\left(\frac{1}{2}-m_{1}\right)(1-m) n \\
& \frac{\partial m_{2}}{\partial t}=-k\left|\hat{\mathcal{F}}_{1}\right| m_{2}+\epsilon\left(\frac{1}{2}-m_{2}\right)(1-m) n .
\end{aligned}
$$

Analysis of the solutions in $\left(m_{1}, m_{2}\right)$-phase space reveals that, although a continuum of equilibria exist, there is long-time convergence to the particular isotropic solution $m_{1}=m_{2}=\frac{1}{2}$ (details not shown). Figure 11 confirms that flux-induced alignment is a transient phenomenon in this model, but also that ECM reorganization to the isotropic state occurs over a long time scale. Near the original wound boundary, ECM alignment is effectively permanent.

Clinical observations of excessive anisotropy and (possibly resultant) mechanical stress near the periphery of a healed scar (Rudolph et al., 1992), together with other features discussed above suggest the scenario depicted in Figure 11 may be the most biologically realistic.
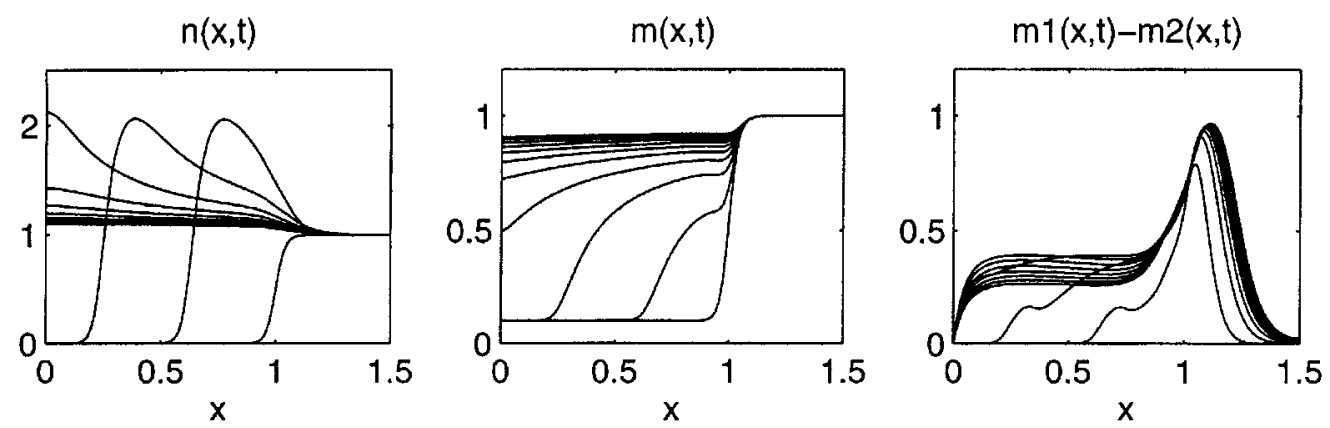

FIGURE 11 Numerical solution of equations (10) together with (20) and (21) with parameter values and end-conditions as in Figure 5, except $\epsilon=1$. This change in $\epsilon$ confirms that the negative feedback in equations (20) and (21) effectively decelerates ECM accumulation in the wound: had $\epsilon=0.2$ been used (as in Figure 5), then ECM accumulation would have been markedly slower. Over a much longer time scale, $m_{1}-m_{2}$ relaxes back towards zero, showing that ECM alignment is transient. Around the wound margins, where considerable alignment occurs, this time scale is so long that alignment is effectively permanent. 


\section{SUMMARY AND DISCUSSION}

\subsection{Summary}

Following a biological introduction to ECM fibre alignment and its importance in dermal wound healing (Section 1), we have presented a relatively simple method of incorporating the key processes of cell flux-induced alignment and contact-guidance feedback into a mathematical model for ECMmediated wound healing (Section 2). This is the first attempt to mechanistically describe the causes and effects of ECM fibre alignment in wound healing in relation to cell-ECM interactions. Numerical simulations of the model in one and two spatial dimensions are presented, showing that throughout most of the domain, the spatiotemporal dynamics (and the coupled alignment responses) are effectively onedimensional.

In the model. the alignment rate parameter $k$ essentially measures the sensitivity of the alignment response to the local cell flux. A key issue is the dependence of the long-term degree of fibre alignment on key parameters such as $k$. Analysis of the model in 1-D reveals that the speed of healing increases with $k$ due to contact-guidance effects, but depends strongly on the values of the cell proliferation and cell motility coefficients. Moreover. numerical simulations suggest that the degree of steady-state alignment increases with $k$ and with the speed of healing, reinforcing the view that faster healing is compromised by severe scarring (as measured by the degree of ECM fibre alignment).

Analysis of the travelling wave structure of the model shows that the steady-state degree of alignment not only increases with $k$, but also with the square-roots of the cell motility and proliferation coefficients. These results are obtained via several approximations which lead to a considerably simpler problem than that of the full partial differential equation model. Despite its being an approximation, this simpler model is effective at quantitatively predicting the steady-state alignment.

The above modelling framework enables us to also analyse the role of contact-guidance. The model can be easily modified to the case where there is no contact-guidance feedback effect. Similar analysis to that presented in Section 2 can be carried out and reveals that contact-guidance increases the speed of healing and enhances fibre alignment. Within this modelling framework one can explore the role of multiple cell and matrix types (Section 3) and investigate the effect of biosynthesis and the permanance of matrix alignment (Section 4).

The model presented in this paper considers only flux-induced alignment. However, cells exert contraction forces on the ECM and thus fibrealignment can be induced via stress. This requires a different modelling framework as the mechanical aspects of cell-matrix interaction need to be explicitly included. In a subsequent publication, we address this type of stress-induced fibre alignment (Olsen et al., 1998).

Experimental data on the spatiotemporal patterns of fibrillar ECM alignment, together with the model predictions described in this paper, may yield new insight into how cellular motility and ECM alignment are coordinated during wound healing. Incorporating our modelling approach into more detailed wound healing models will enable specific predictions to be made on the potential to regulate ECM alignment, which would have important therapeutic implications.

\section{Acknowledgement}

We thank John Dallon, Eamonn Gaffney and Mark Ferguson for helpful discussions. L. O. expresses gratitude to the Engineering and Physical Sciences Research Council (EPSRC) of Great Britain for a Earmarked Research Studentship award to support this work and B. M. thanks the EPSRC for a Pool Research Studentship award. JAS and PKM were supported in part by grant GR/K71394 from the EPSRC. We also thank the London Mathematical Society for support under Scheme 3.

\section{References}

Birk, D. and Trelstad, R. (1986) Extracellular compartments in tendon morphogenesis: collagen fibril, bundle and microaggregate formation. J. Cell Biol., 103, 231-240. 
Chaplain, M. A. J. and Byrne, H. M. (1996) Mathematical modelling of wound healing and tumour growth -2 sides of the same coin. Wounds: A Compendium of Clinical Research and Practice, 8, 42-48.

Civelekoglu, G. and Edelstein-Keshet, L. (1994) Modelling the dynamics of $f$-actin in the cell. Bull. Math. Biol., 56, 587-616.

Clark, R. A. F. (1988) Overview and general considerations of wound repair. In Clark, R. A. F. and Henson, P. M., editors, The Molecular and Cellular Biology of Wound Repair, Plenum, New York, chapter 1, pages 3-34.

Clark, R. A. F. (1993) Biology of dermal wound repair. Dermatol. Clin., 11, 647-666.

Cook, J. (1995) Waves of alignment in populations of interacting, oriented individuals. Forma, 10, 171-203.

Deutsch, A. (1995) Towards analysing complex swarming patterns in biological systems with the help of lattice-gas cellular automata. J. Biol. Systems, 3, 947-956.

Dickinson, R. B. and Tranquillo, R. T. (1993) A stochastic model for adhesion-mediated cell random motility and haptokinesis. J. Math. Biol., 31, 563-600.

Eastwood, M., McGrouther, D. A. and Brown, R. A. (1994) A culture force monitor for measurement of contraction forces generated in human dermal fibroblast cultures - evidence for cell-matrix mechanical signalling. Biochim. Biophys. Acta, 1201, 186-192.

Edelstein-Keshet, L. and Ermentrout, G. (1990) Models for contact-mediated pattern formation: cells that form parallel arrays. J. Math. Biol., 29, 33-58.

Ehrlich, H. P. and Rajaratnam, J. B. M. (1990) Cell locomotion forces versus cell contraction forces for collagen lattice contraction: An in vitro model of wound contraction. Tiss. Cell. 22, 407-417

Fung, Y. C. (1993) Biomechanics. Springer-Verlag, New York, second edition.

Geigant, E., Ladizhansky, K. and Mogilner, A. (1997) An integrodifferential model for the orientational distribution of $f$-actin in cells. preprint.

Grinnell, F. (1992) Cell adhesion. In Cohen, I. K., Diegelmann, R. F., and Lindblad, W. J., editors, Wound Healing: Biochemical and Clinical Aspects, Saunders, Philadelphia; London, chapter 12, pages 209-222.

Grunbaum, D. (1997) Advection-diffusion equations for generalised tactic searching behaviors. J. Math. Biol., [in press].

Guidry, C. and Grinnell, F. (1992) Studies on the mechanism of hydrated collagen gel reorganization by human skin fibroblasts. J. Cell Sci., 79, 67-81

Harmon, C. B., Zelickson, B. D., Roenigk. R. K., Wayner, E. A., Hoffstrom, B., Pittelkow, M. R. and Brodland, D. G. (1995) Dermabrasive scar revision - immunochemical and ultrastructural evaluation. Dermatol. Surg., 21, 503-508.

Harris, A. K., Wild, P. and Stopak, D. (1980) Silicone rubber substrata: A new wrinkle in the study of cell locomotion. Science, 208, 177-179.

Ingber, D. and Folkman, J. (1988) Inhibition of angiogenesis through modulation of collagen metabolism. Lab. Invest., 59 44-51.

Mast, B. A. (1992) The skin. In Cohen, I. K., Diegelmann, R. F and Lindblad, W. J., editors, Wound Healing: Biochemical and Clinical Aspects, Saunders, Philadelphia; London, chapter 22, pages $344-355$

McCarthy, J. B., Sas, D. F. and Furcht, L. T. (1988) Mechanisms of parenchymal cell migration into wounds. In Clark, R. A. F. and Henson, P. M., editors, The Molecular and Cellular Biology of Wound Repair, Plenum, New York, chapter 13, pages $281-319$
McPherson, J. M. and Piez, K. A. (1988) Collagen in dermal wound repair. In Clark, R. A. F. and Henson, P. M., editors, The Molecular and Cellular Biology of Wound Repair, Plenum, New York, chapter 20, pages 471-496.

Mogilner, A. and Edelstein-Keshet, L. (1996) Spatio-angular order in populations of self-aligning objects: formation of oriented patches. Physica D, 89, 346-367.

Murray, J. D. (1993) Mathematical Biology. Springer-Verlag, New York.

Olsen, L., Maini, P. K., Sherratt, J. A. and Arnold, F. (1997) A mathematical model for the capillary endothelial cellextracellular matrix interactions in wound-healing angiogenesis. IMA J. Math. Appl. Med. Biol, 14, 261-281.

Olsen, L., Sherratt, J. A. and Maini, P. K. (1995) A mechanochemical model for adult dermal wound contraction and the permanence of the contracted tissue displacement profile. $J$ theor. Biol., 177, 113-128.

Olsen, L., Sherratt, J. A. and Maini, P. K. (1996) A mathematical model for fibro-proliferative wound healing disorders. Bull. Math. Biol., 58, 787-808.

Olsen, L., Maini, P. K., Sherratt, J. A. and Dallon, J., Mathematical modelling of anisotropy in fibrous connective tissue (submitted).

Peacock, E. E. (1984) Wound Repair. Saunders, Philadelphia.

Perumpanani, A. J., Sherratt, J. A., Norbury, J. and Byrne. H. M (1996) Biological inferences from a mathematical model for malignant invasion. Imvasion and Metastasis, 16, 209-221

Pettet, G. J., Byrne, H. M., McElwain, D. L. S. and Norbury, J. (1996) A model of wound-healing angiogenesis in soft tissue. Math. Biosci., [in press].

Repesh, L. A., Fitzgerald, T. J. and Furcht, L. T. (1982) Fibronectin involvement in granulation tissue and wound healing in rabbits. J. Histochem. Cytochem., 30, 351-358.

Rudolph, R.. Berg, J. V. and Ehrlich, H. P. (1992) Wound contraction and scar contracture. In Cohen, I. K. Diegelmann, R. F. and Lindblad, W. J., editors, Wound Healing: Biochemical and Clinical Aspects, Saunders, Philadelphia; London, chapter 6 . pages $96-114$.

Ryan, T. J. (1989) Biochemical consequences of mechanical forces generated by distention and distortion. J. Am. Acad. Dermatol., 21, 115-130.

Shah, M., Foreman, D. M. and Ferguson, M. W. J. (1992) Control of scarring in adult wounds by neutralising antibody to transforming growth factor $\beta$. Lancet, 339, 213-214.

Sherratt, J. A. (1994) Chemotaxis and chemokinesis in eukaryotic cells: The Keller-Segel equations as an approximation to a detailed model. Bull. Math. Biol., 56, 129-146.

Sherratt, J. A. and Lewis, J. (1993) Stress-induced alignment of actin filaments and the mechanics of cytogel. Bull. Math. Biol. 55, $637-654$

Stevens, A. (1995) Trail following and aggregation of myxobacteria. J. Biol. Systems, 3, 1059-1068.

Stopak, D. and Harris, A. K. (1982) Connective tissue morphogenesis by fibroblast traction. I: Tissue culture observations. Dev. Biol., 90, 383-398.

Tracqui, P., Woodward, D. E., Cruywagen, G. C., Cook, J. and Murray, J. D. (1995) A mechanical model for fibroblast-driven wound healing. J. Biol. Systems, 3, 1075-1084.

Tranquillo, R. T. and Murray, J. D. (1992) Continuum model of fibroblast-driven wound contraction: Inflammation-mediation. J. theor. Biol., 158, 135-172.

Trinkaus, J. P. (1984) Cells into Organs. The Forces that Shape the Embrvo. Prentice-Hall, Englewood Cliffs. N. J.

Vernon, R. B., Angello, J. C., Iruela-Arispe, M. L., Lane, T. F. and Sage, E. H. (1992) Reorganization of basement membrane 
matrices by cellular traction promotes the formation of cellular networks in vitro. Lab. Invest., 66, 536-547.

Vernon, R. B. and Sage, E. H. (1995) Between molecules and morphology. Extracellular matrix and creation of vascular form. Am. J. Pathol., 147, 873-883.

Weinberg, C. B. and Bell, E. (1985) Regulation of proliferation of bovine aortic endothelial cells, smooth muscle cells and adventitial fibroblasts in collagen lattices. J. Cell. Physiol., 122, 410-414
Welch, M. P., Odland, G. F. and Clark, R. A. F. (1990) Temporal relationships of $F$-actin bundle formation, collagen and fibronectin matrix assembly, and fibronectin receptor expression to wound contraction. J. Cell Biol., 110, 133-145.

Yoshizato, K., Taira, T. and Yamamoto, N. (1985) Growth inhibition of human fibroblasts by reconstituted collagen fibrils. Biomed. Res., 6, 61-71. 


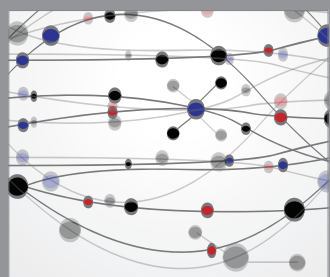

The Scientific World Journal
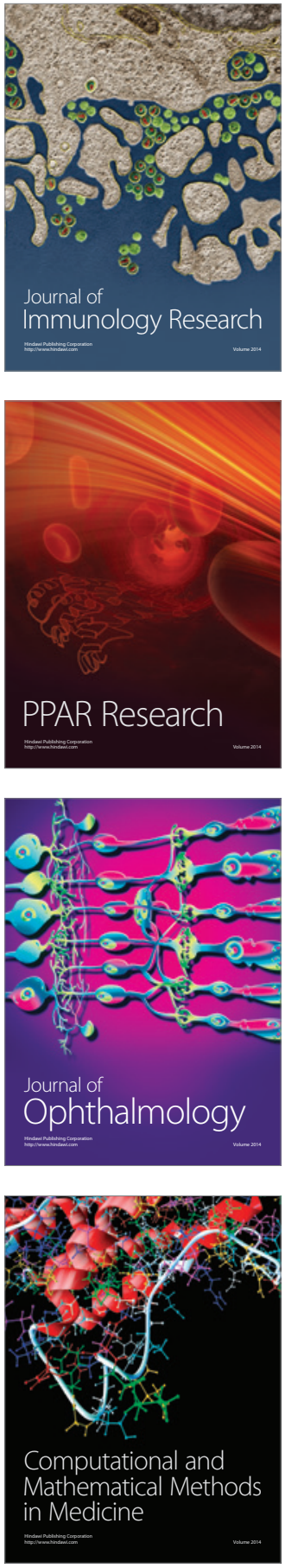

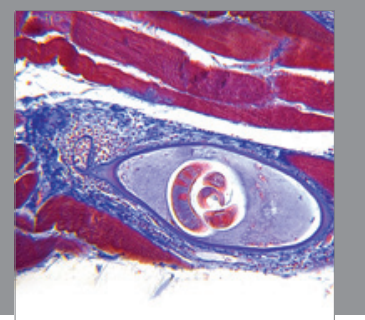

Gastroenterology

Research and Practice
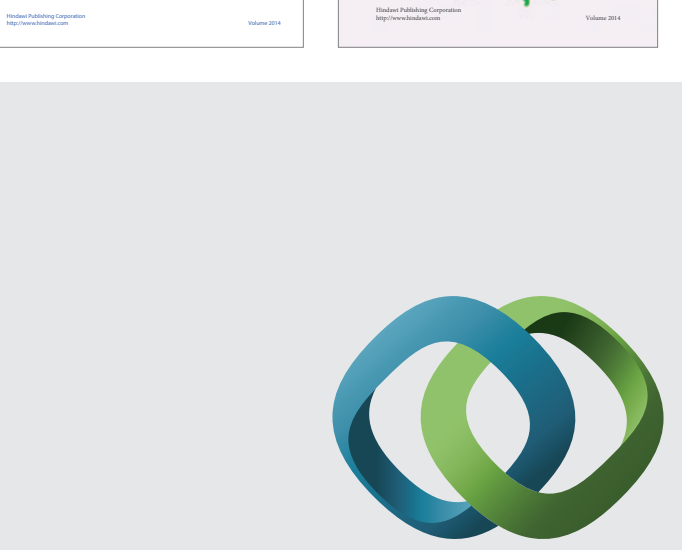

\section{Hindawi}

Submit your manuscripts at

http://www.hindawi.com
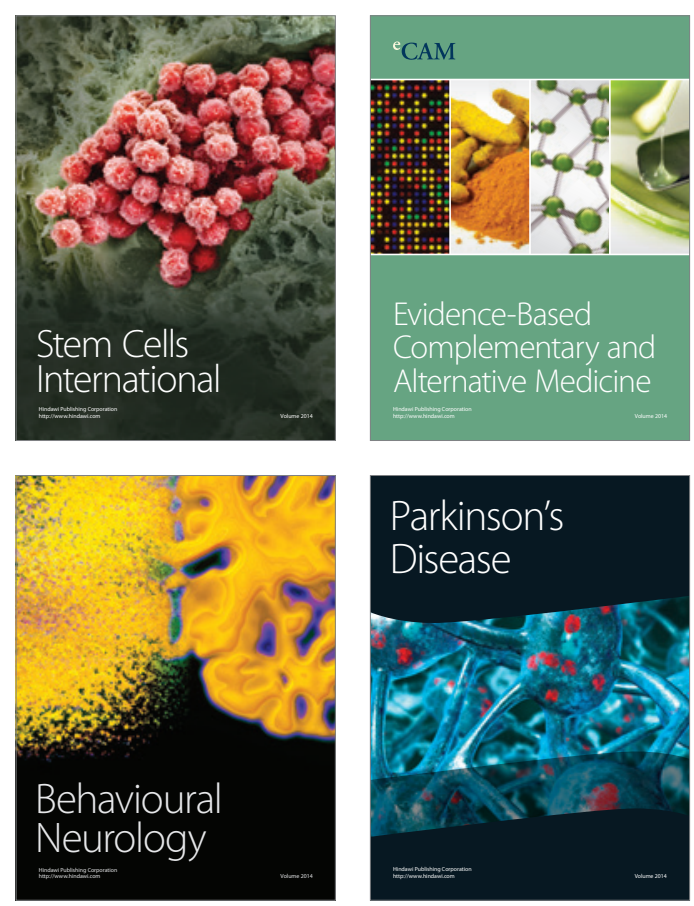

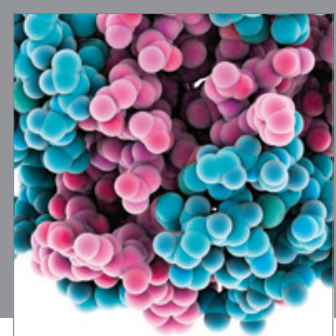

Journal of
Diabetes Research

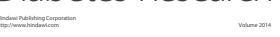

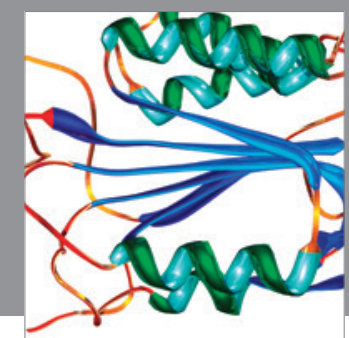

Disease Markers
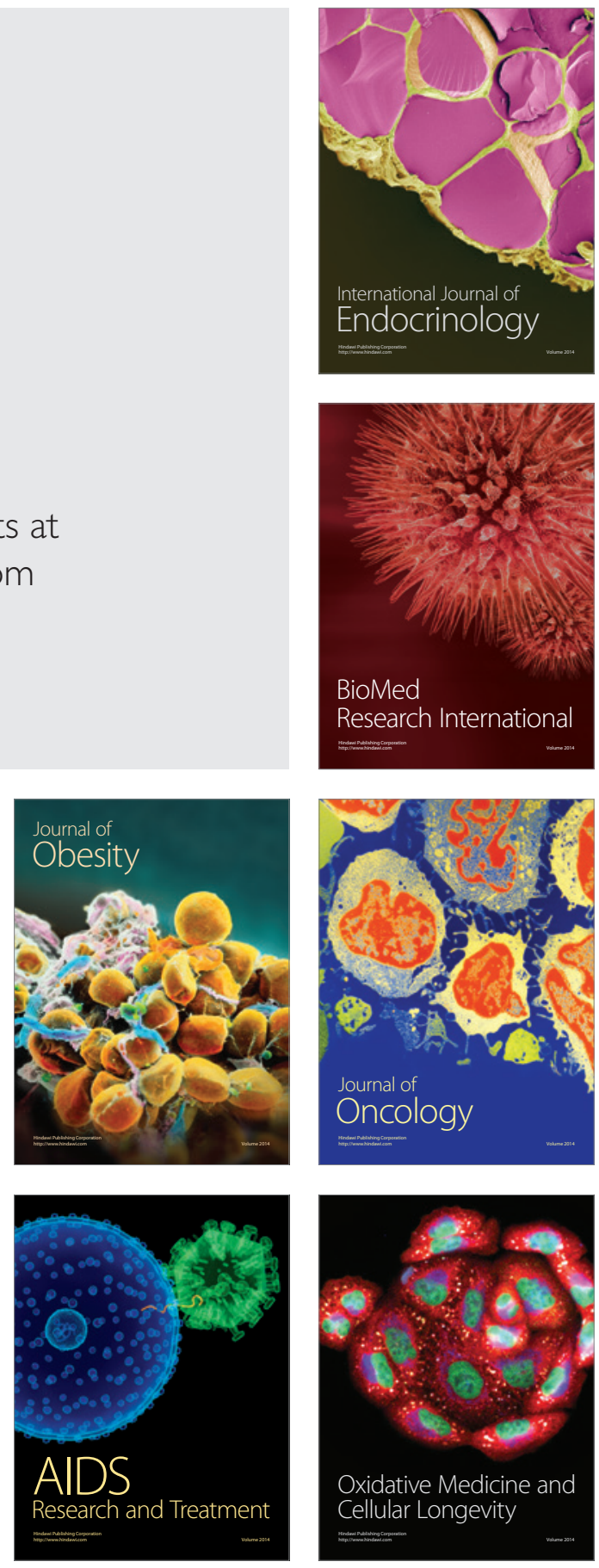